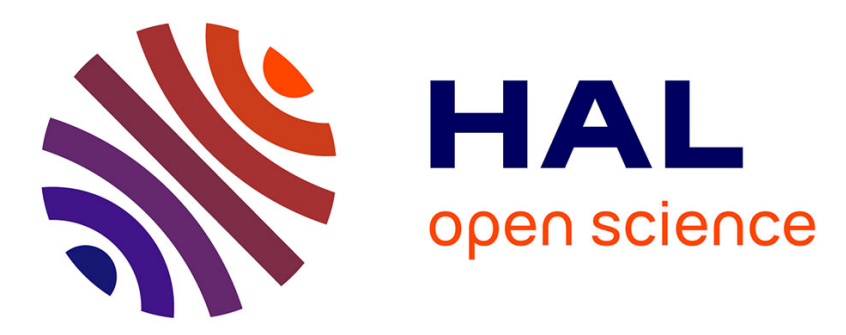

\title{
Mechanical properties of random arrays of short carbon fibers
}

\author{
J. Vareille, E. Guyon, S. Roux
}

\section{To cite this version:}

J. Vareille, E. Guyon, S. Roux. Mechanical properties of random arrays of short carbon fibers. Revue de Physique Appliquée, 1987, 22 (11), pp.1361-1369. 10.1051/rphysap:0198700220110136100 . jpa00245686

\section{HAL Id: jpa-00245686 https://hal.science/jpa-00245686}

Submitted on 1 Jan 1987

HAL is a multi-disciplinary open access archive for the deposit and dissemination of scientific research documents, whether they are published or not. The documents may come from teaching and research institutions in France or abroad, or from public or private research centers.
L'archive ouverte pluridisciplinaire HAL, est destinée au dépôt et à la diffusion de documents scientifiques de niveau recherche, publiés ou non, émanant des établissements d'enseignement et de recherche français ou étrangers, des laboratoires publics ou privés. 
Classification

Physics Abstracts

$64.40 \mathrm{~A}-81.20 \mathrm{~N}-82.70$

\title{
Mechanical properties of random arrays of short carbon fibers $\left(^{*}\right)$
}

\author{
J. Vareille (**), E. Guyon and S. Roux \\ LHMP, Unité associée au CNRS n 857, ESPCI, 10 rue Vauquelin, 75231 Paris Cedex 05, France
}

(Reçu le 7 avril 1987, accepté le 28 juillet 1987)

\begin{abstract}
Résumé. - Les propriétés mécaniques de composites de carbone à fibres courtes ont été étudiées expérimentalement en relation avec leurs propriétés électriques dans les 3 cas suivants : 1) fibres dans une matrice epoxy rigide ; 2) fibres dans un gel bien formé ; 3) fibres sédimentant dans un liquide visqueux. Bien qu'il n'y ait pas de corrélation précise entre le seuil de percolation électrique et l'augmentation continue du module élastique avec la concentration de fibres, on peut voir une relation de plus en plus proche entre ces deux notions lorsqu'on passe de la situation 1 à 3, c'est-à-dire quand l'influence de la matrice décroit. Une discussion portant sur la relation avec les problèmes d'enchevètrements et sur la percolation mécanique est présentée.
\end{abstract}

\begin{abstract}
The mechanical properties of random short carbon fiber composites have been studied experimentally in relation with the electrical properties (continuity) in three different states : 1) fibers in a hard expoxyde matrix, 2) fibers in a well formed gel, 3) fibers sediment in a viscous liquid. Although there is no precise correlation between the electrical percolation threshold and the continuous increase of elastic modulus with fiber concentration, the variation of linear elastic properties can be seen to be more and more related to the connectivity (and possibly entanglement) transition when going from state 1 to 3 , i.e. as the influence of the matrix decreases. A related discussion dealing with entanglement properties and mechanical percolation is presented.
\end{abstract}

\section{Introduction.}

Studies of synthetic fiber composites have undergone important developments in the last two decades associated with the fast increase of their applications. On the theoretical level, methods initially introduced to describe the electric properties of composite mixtures have been applied with some success to these materials : homogeneization techniques (for long fiber oriented composites), self-consistent methods, bounds [1]. These methods, in order to give any valuable information, need to assume periodicity or, at least, regularity in the packing of fibers which is generally the case in long fibers composites. This is clearly not the case in chopped fiber composites made of a tridimensional random and tenuous net of entangled fibers. This class of materials has undergone important recent develop-

$\left(^{*}\right)$ A detailed presentation can be found in J. Vareille Thèse $3^{\mathrm{e}}$ cycle, Paris VI (1986).

(**) Permanent address : ENSIETA, rue F. Verny, 29240 Brest Naval, France. ments in the fabrication of sheet molding compounds. They can be processed and can be prepared at low cost and involve large concentrations of fibers. We will consider only the limit of low concentration of short fibers. Another application which has stimulated fundamental research on random packings of fibers [2] is the fabrication of videodiscs. In particular, around the concentration where the fibers start forming a continuous structure (percolation threshold), there are singular geometric fluctuations of statistical origin which add up to possible heterogeneities created in the preparation.

In this article we describe a series of mechanical experiments involving a random array of conductive graphite fibers :

- the first experiments use fibers contained in an epoxyde matrix. The results are compared with a joint study of electrical properties of the same materials by Carmona et al. [3]. However the matrix is too " hard » to allow for the observation of any effect related to the continuity of the stack of fibers,

- the second set of experiments uses fibers 
contained in a well-formed polyacrylamide soft gel. There is indication of nonlinear variation of the elastic modulus in the range of fiber concentration where electrical percolation takes place,

- finally, we consider a sediment bed made of entangled fibers which have sedimented on the bottom of a cylindrical vessel starting from an initially stirred suspension of fibers.

In the experiments, we can vary the length $L$ of the initially monodisperse fibers as well as the concentration (in the two first sets of experiments). However these parameters are insufficient to fully characterize the entanglements which should depend on the fiber-fiber and fiber-matrix interface, on the fluid in which the fibers are initially mixed (initial viscosity) and on the characteristics of the flow (as is also well-known for a polymer fiber processing). The present results should be considered as preliminary and the analysis suggestive of possible approaches of the mechanics of such heterogeneous packings.

Section 2 contains a discussion of the preparation of the three classes of samples. In section 3 to 5 the results on the experiments are presented and discussed. Percolation, which has been very effective to describe the «non homogeneizable » electrical properties of composites near certain concentration thresholds, is reviewed in the appendix.

\section{Preparation and structure analysis.}

\subsection{SAMPLE PREPARATION.}

2.1.1 Fiber + resin. - We have used chopped long bundles of $8 \mu \mathrm{m}$ diameter graphite fibers made by Carbone-Lorraine using an array of parallel razor blades which could produce monodisperse fibers of length $L$ varying between 1.1 and $9.8 \mathrm{~mm}$. The matrix was an epoxyde resin polymerizable at room temperature. The components were intimately mixed and then moulded in teflon containers. The reaction of polyaddition took place at a regulated temperature $\left(55^{\circ} \mathrm{C}\right)$ under nitrogen atmosphere. After polymerization, samples were cut on a milling machine in the form of parallelepipeds.

2.1.2 Fibers + gels. - We have used the same kind of fibers as above $(L=1.1 \mathrm{~mm})$. The matrix was a polyacrylamide gel obtained by copolymerization of mono- and bisacrylamide in the presence of an activator (potassium persulfate) and of a basic accelerator of reaction (tetramethyldiamine) [4]. The components were introduced in polystyrene molds of size $50 \times 50 \times 10 \mathrm{~mm}$ or in cylindrical tubes of glass $(L=50 \mathrm{~mm}$, width $11 \mathrm{~mm})$. The components were permanently stirred in order to maintain a uniform concentration, as checked visually, up to formation of the gel. The gels were subsequently taken out of the mold and cut into parallelepipedic $(15 \times 10 \times 10 \mathrm{~mm}$ typically $)$ or cylindrical shape respectively. As no chemical coating of the fibers was used, these are simply held within the gel.

2.1.3 Problems in sample preparations. - We have not been able to prepare homogeneous samples with individual fibers when the concentration was too large (typically $\Phi \geq 1 \%$ for $L=3 \mathrm{~mm}$ ). For larger concentrations, the fibers aggregated in oriented bundles or in pin-cushions. This also led to appreciable breaking of the fibers and to a final polydisperse distribution of fiber lengths (up to $80 \%$ of broken fibers).

2.2 ON THE RANDOM PACKING OF FIBERS. - Even in the simplest case of the packing of monodisperse spheres, there is a large number of possible packed states characterized by their filling factor $(1-\Phi$; where $\Phi$ is the porosity), average number of contacts (or coordinance), and short range order characterized by radial (and angular for elongated objects) correlation function. These states depend on the packing conditions, on the subsequent cycles of pressure, on the interfacial conditions as characterized by the roughness and the Coulomb limit friction angle (between normal and tangential component of forces).

A powerful approach for the packing of non space filling objects like fibers (spheres are space filling) uses the concept of excluded volume introduced by Onsager for the isotropic-nematic transition of long rigid molecules (e.g. Tobacco Mosaic Virus) [5]. It is defined as the volume in which the centre of an object must lay in order for it to overlap a second fixed object averaged overall possible relative orientations, and can be obtained for a variety of geometries by classical theorems of Integral Geometry [6].

In the case of rods of length $L$ and radius $r$ interacting as hard core objects randomly oriented in space, it is equal to :

$$
V_{\mathrm{ex}}=\pi L^{2} R+\mathrm{o}\left(L R^{2}\right)
$$

In Onsager thermodynamic treatment, $V_{\text {ex }}$ enters as a second order correction in a virial expansion of the free energy. There is a first order transition to a Nematic with a finite long range order in the orientations for a critical value of the number of fibers per unit volume such that :

$$
N_{\mathrm{cN}} V_{\mathrm{ex}}=\text { Const } \text {. }
$$

Note that this concentration is very small (when $R / L \ll 1$ ) compared to that for packing. More recently, the same concept has been applied to the determination of the percolation threshold of conducting objects [2].

The quantity $N_{\mathrm{c}} V$ can be understood as an average number of contacts per object which is known to be nearly constant (quasi-invariant) for various bond percolation problems and which is such 
that $N_{\mathrm{cp}} V_{\mathrm{ex}} \sim 1.4$ in the particular case of the percolation threshold of long rods. This result was confirmed on various numerical experiments which gave the conjected dependence of $N_{\mathrm{cp}}$ in $R^{-1} L^{-2}$, or the critical volume fraction $\Phi_{\mathrm{c}} \sim N_{\mathrm{cp}}\left(L R^{2} \pi\right)$ as $\Phi_{\mathrm{c}} \sim 1.4(R / L)$.

The existence of various thresholds given by the same dimensionless form shows the multiplicity of macroscopic configurations of the packing with an ordered (crystalline) phase and a multiplicity of metastable entangled (glassy) phases.

We may expect that the random lattice of rods is not rigid above the concentration $N_{\mathrm{cp}}$ but only above a higher entanglement threshold about which we can only formulate a few conjectures :

we can assume that the only difference between this higher threshold and the continuity one would be in the larger number of contacts needed to reach rigidity. If we let fibers fall one after the other on the top of a sedimenting bed (see 3.3) without reorganisation of the packing, we may expect each new fiber to stop when it has established a number of contacts corresponding to the nature of these contacts. However the importance of the quality or nature of contacts (e.g. existence and importance of friction) is of the uttermost relevance: in stabilizing the glassy phase and in determining the critical volume fraction $\Phi_{\mathrm{c}}$. Indeed, the number of contacts $N_{\mathrm{cp}} V_{\mathrm{ex}}$ must take into account the number of degrees of freedom blocked by a contact.

\section{Mechanical studies of composites.}

3.1 RESIN + FIBERS. - The mechanical study was carried out in parallel with an electrical study of the same system initiated by Carmona et al. [3]. We will first review briefly these electrical studies and will present our mechanical results.

\section{- Electrical results.}

A series of samples having a given fiber length $L$ shows an electrical percolation threshold as the value of the concentration of fibers exceeds a critical value $\Phi_{\mathrm{c}}(L)$. The properties of the transition from the insulating state - insulating epoxyde matrix to a conducting one are recalled in the appendix.

The results are given in figure 8 as a function of the ratio $L / d$ where $d$ is the diameter of the rod. On the same plot we have indicated the results of numerical simulations by Balberg and Binenbaum [2] on randomly oriented cylinders in all directions of space and those of Carmona [3] using only cylinders along 3 orthogonal directions. The slope -1 is that deduced from the excluded volume argument given in the previous chapter. The experiments only qualitatively agree with this fit. This is probably indicative of the fact that, as the length of the fibers changes, the action of the mixing may lead to different packings. However there is a clear indica- tion of an electrical percolation behaviour. The value of the threshold being strongly related to the entanglements produced during the mixing.

\section{- Mechanical results.}

We have submitted the samples to compression, traction and flexion; we have also studied the impact on strength and relaxation characteristics. The last experiments did not show any significant variation in amplitude when $\Phi$ varies, suggesting that the mechanical behaviour is always governed by that of the matrix. We will only consider the traction-compression results. In figure $1 \mathrm{a}$ and $\mathrm{b}$ the stress-strain relation given on two samples of the same composition ( $\Phi=0.3 \%$; average length of fibers $\langle L\rangle=1.1 \mathrm{~mm}$ ). In the traction experiment there is an appreciable intermediate linear regime which was used for the determination of the Young's modulus. Beyond the maximum stress, a domain of plastic deformation is observed with striction and, finally, ductile rupture. The striction becomes more pronounced as the fiber concentration decreases.

On compression curves, a simple linear behaviour is observed from the origin on. The rupture takes place after a long domain of plastic deformation. The order of magnitude of the modulus is large compared to that of the second series of experiments with a gel matrix, indicating that the direct contribution of the fiber lattice is small with respect to that due to the epoxyde and to the gel-epoxyde interaction.

In figure 3 , we have plotted the elasticity results for both compression and traction as a function of the fiber concentration for the same set of fibers as in figure 2. The crosses give averages and the squares give a value obtained by extensometric method. The upper and lower curves are the Voigt and Reuss first order bounds (Hashin [1]) which correspond to series and parallel arrangments of the phases.

There is no indication of anomalies around the volume fraction $\Phi_{\mathrm{c}}=1.5 \%$ where the electrical measurements for the same length $L=1.1 \mathrm{~mm}$ of fibers indicate a percolating transition to a conducting state. This is not surprising because of the small rigidity of the structure of fibers which does not contribute much to the total rigidity. However, we have observed some qualitative changes of the non linear mechanical properties and as $\Phi$ varies across this range : as the concentration of fibers increases from a few percent, rupture turns from a ductile to a brittle type.

3.2 GEL + FIBERS. - We are dealing here with a well formed gel well above gelation threshold where there is a much larger contrast between both phases. Consequently we expect effects related to the change in the geometry of the fiber assembly with the concentration. 

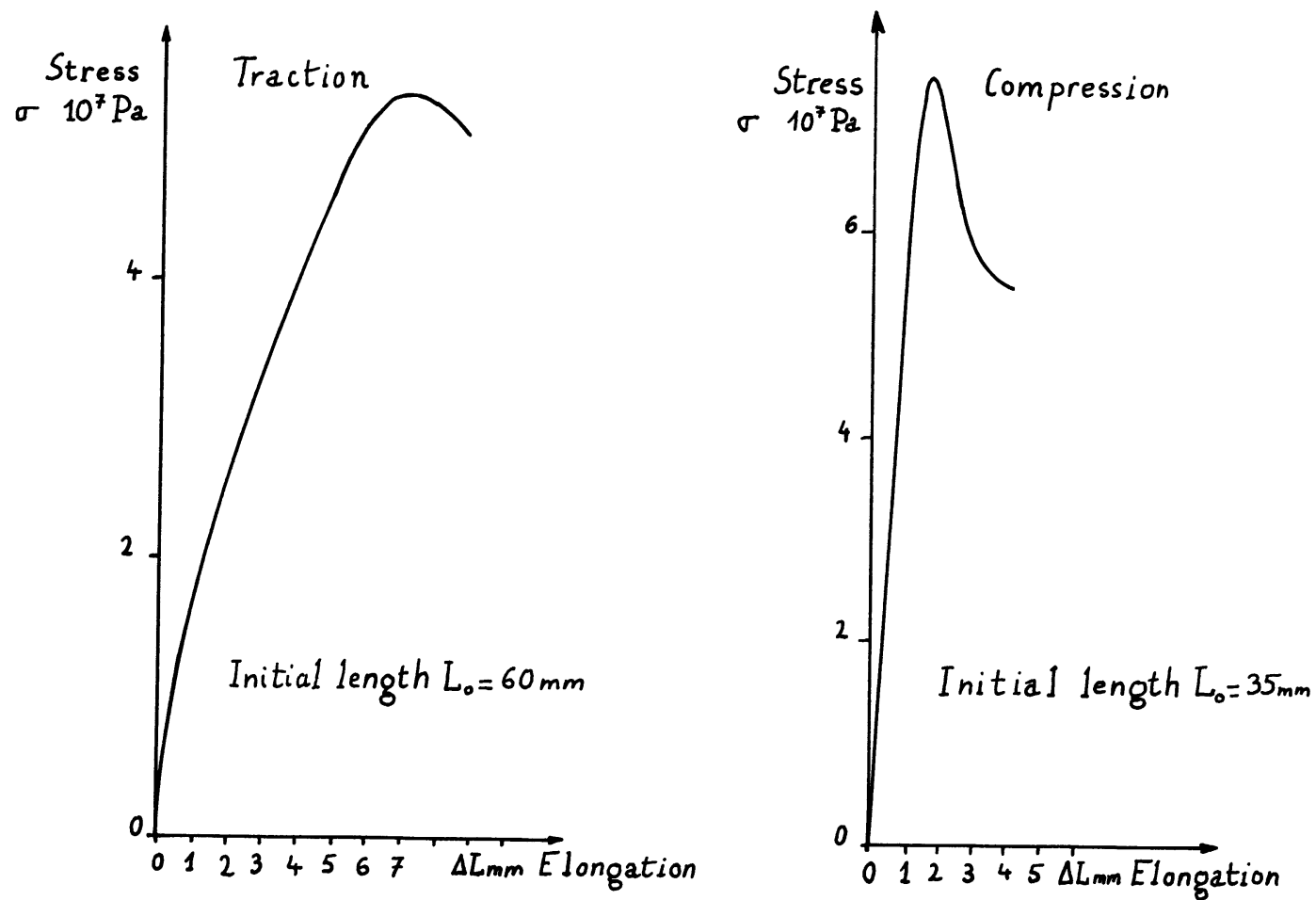

Fig. 1. - Typical stress-strain relation for traction and compression of an epoxyde matrix reinforced with $0.3 \%$ of short carbon fibers.

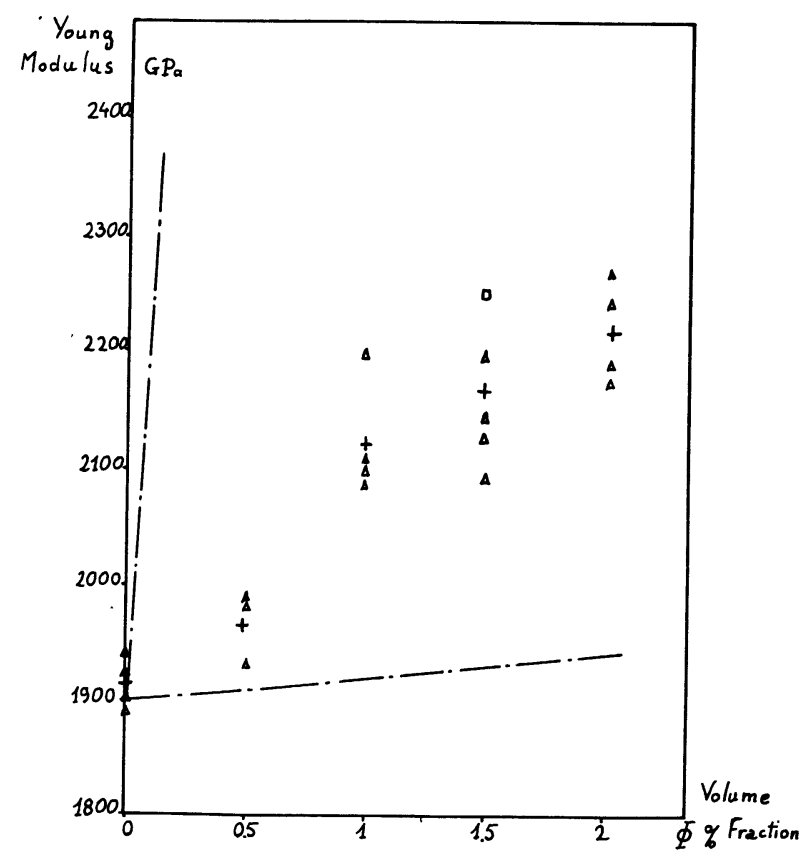

Fig. 2. - Evolution of the Young modulus for compression of an epoxyde matrix charged with short carbon fibers. The abscissa is the volume concentration of fibers. Let us note that no singular behaviour is visible around the percolation threshold $(1.5 \%)$.

In order to be able to measure the weak elasticity of these samples $\left(10^{-4} \mathrm{~N} / \mathrm{m}^{2}\right)$ we have constructed an automatic elasticity-micropress mounted on a scale which could be programmed in displacement

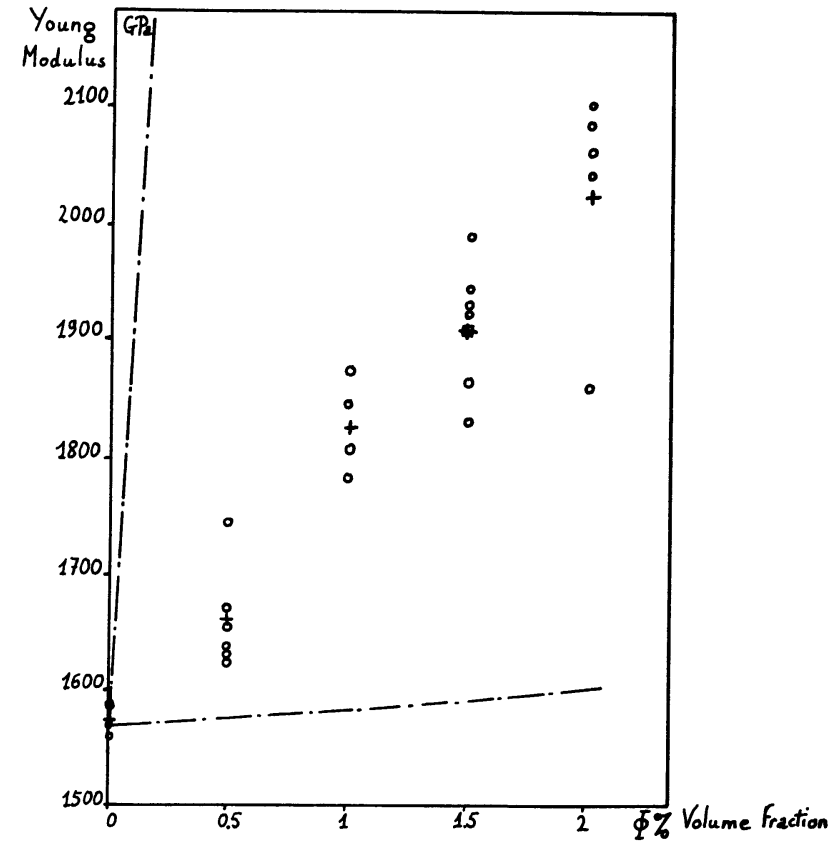

Fig. 3. - Same as figure 2 for traction.

amplitude to give a complete stress-strain curves. This press will be described in an independent article (Vareille [7]).

Preliminary experiments on pure gels have shown that :

- it is not necessary to keep the gel in water solution during the experiment. A whole exper- 
imental curve takes only 3 min. A typical relaxation associated with the motion of water out of the gel becomes perceptible typically after $15 \mathrm{~min}$,

- deformation takes place at constant volume,

- on pure gels, even for large deformations (more as $50 \%$ ) there is only a weak hysteresis,

- similar features are obtained on gel-fibers composites as for pure gels apart from the existence of a long hysteresis.

Figure 4 gives a stress strain relation for a composite whose physical parameters are $\Phi=1.5 \%$, $L=1.1 \mathrm{~mm}$. The stress is defined as the ratio of the applied force to the surface at this stage of compression. The surface is calculated by expressing that the gel is incompressible (Poisson ratio $\nu=1 / 2$ ) which was verified directly. On the horizontal axis of the figure 4 we have plotted $\ln (1+\varepsilon)$ instead of the strain $\varepsilon$. These two variations are equivalent for small $\varepsilon$ but the former one is found to give a much larger range of linear variation with stress for pure gels (Treloar [8] ; this point is discussed in detail in Vareille's thesis [7]).

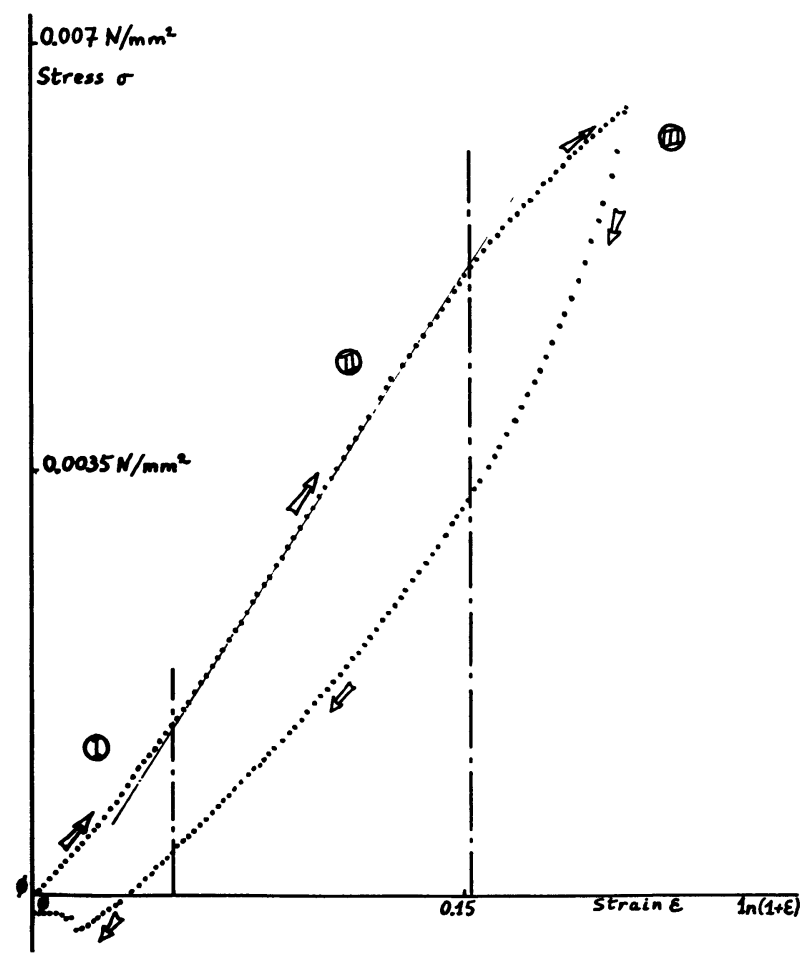

Fig. 4. - Compression of a short-fiber-armed gel (polyacrylamide) (1.5\% of fibers). I : Non-linear behaviour due to geometrical imperfection of the sample. II : Linear stress-strain domain over which the elastic constant is determined. III : Damping of the gel due to too large stresses. This results in an hysteretic return to the origin.

The load curve shows 3 regimes :

- region $I$ in which there is a macroscopic readjustment of the sample geometry,
- region II is a reversible line domain which we have used to calculate the Young's modulus. The domain of linearity decreases as the concentration of fibers increases,

- region III leads to an hysteretic degradation of the sample which appears only on gel-fibers composites.

We have measured the mechanical properties of 32 samples of different concentrations. Afterwards, we have also measured the electrical conductance of a fraction of them. Figure 5 shows a continuous increase of the average compression modulus with $\Phi$. The ratio of the values of the moduli for

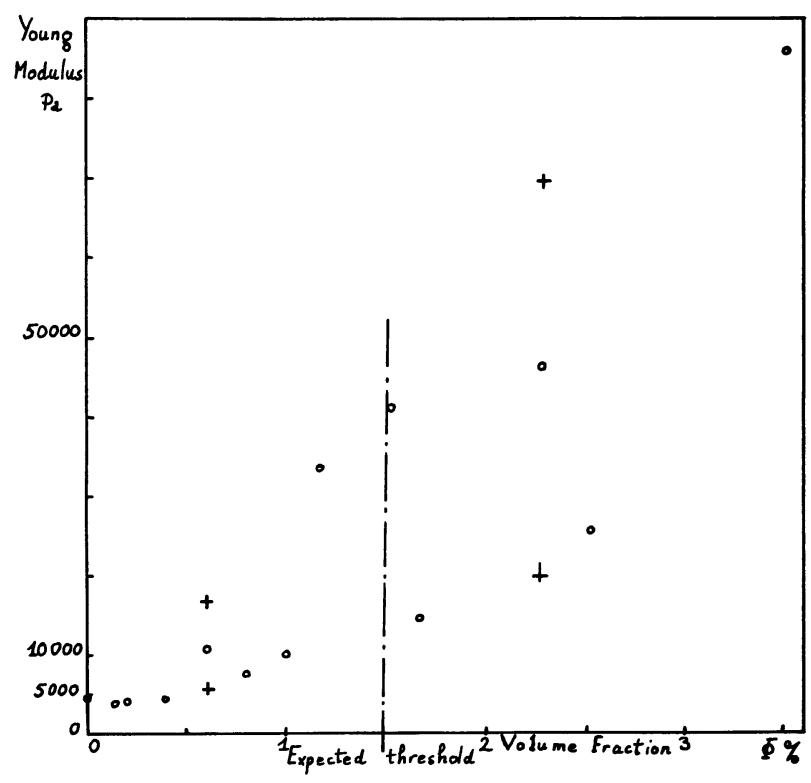

Fig. 5. - For polyacrylamide gels armed with short carbon fibers, the Young modulus is shown versus the volume concentration of fibers. The dashed line shows the expected percolation threshold.

$\Phi=0$ and $3.5 \%$ is of the order of 20. We have also indicated the electrical threshold obtained in epoxyde-fiber composites of same fiber's length. An additional piece of information is given by the fluctuations in the values of modulus obtained for the same concentration. It also increases with concentration with an indication of maximum around $1.5 \%$. The final observation is in figure 6 , where we have plotted the Young's modulus versus resistivity on 8 samples. The 3 circle points correspond to different samples with the same nominal concentration. Despite the dispersion of data, there is a good correlation between the electrical and mechanical data suggesting that the same fluctuations in the fiber packing are responsible for an increase of elastic modulus and decrease of resistivity (and vice versa). 


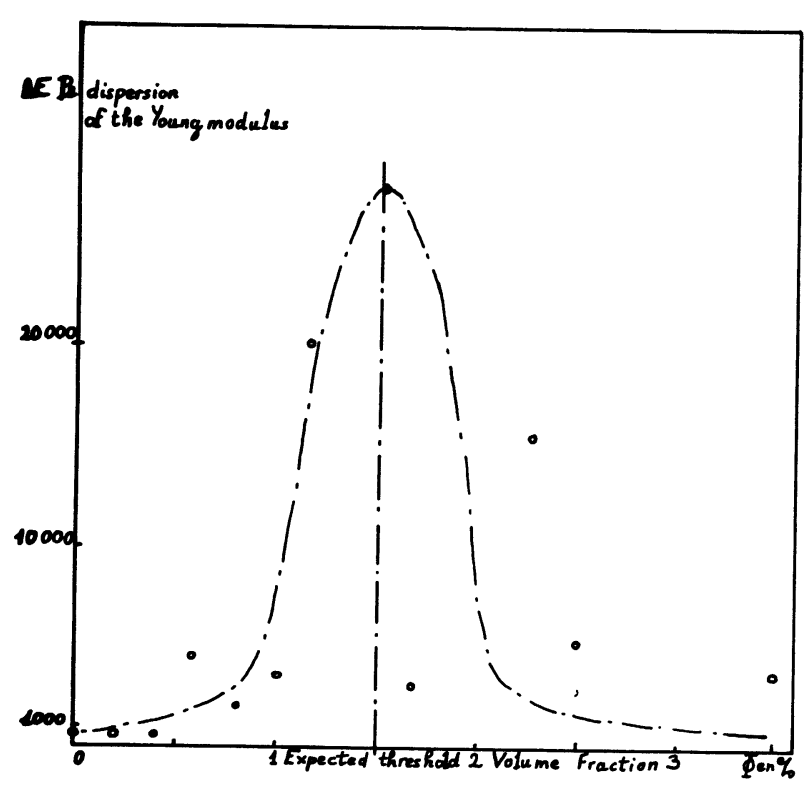

Fig. 6. - Standard mean deviation of the measured values of the Young modulus from figure 5.

It is rather tempting to relate the above results to a percolation transition of the mechanical system. The non linear increase of Young modulus which is clearly visible above $\Phi=1 \%$ could indicate a crossover from a regime dominated by the modulus of the gel $(\Phi=0)$ to one where the formation of a continuous fiber network causes the faster increase of modulus. The maximum in the dispersion of data could be related to a finite size effect. In percolation problems, there is a correlation length which characterizes the size of the representative elementary volume of the composite and which should diverge (typically as $L\left|\left(\Phi-\Phi_{\mathrm{c}}\right) / \Phi_{\mathrm{c}}\right|^{-0.89}$ in 3 dimensions) around percolation threshold $\Phi_{\mathrm{c}}$. An estimate of this length indicates that fluctuations of composition of purely statistical origin due to the finite size $\left(L_{0} \sim 1 \mathrm{~cm}\right)$ of the sample should have a maximum over a range of $30 \%$ of $\Phi_{c}$ around threshold ; this is not unreasonable considering our results. There are probably other fluctuations due to packing heterogeneities which also give rise to local fluctuations in the geometry of the fibers and, hence, reinforce the data dispersion.

The correlation between the evolution of the Young modulus and that of the conductivity indicates that the increase in the number of contact points between fibers which controls the conductivity also controls the elasticity. This does not necessarily imply a correspondance between the electrical threshold and a mechanical one smeared by the effect of the finite gel elasticity (as in the discussion of formula A.5 in the appendix for the corresponding electrical problem). One can also think that there is a mechanical threshold for soft to hard material which would take place for higher concentrations

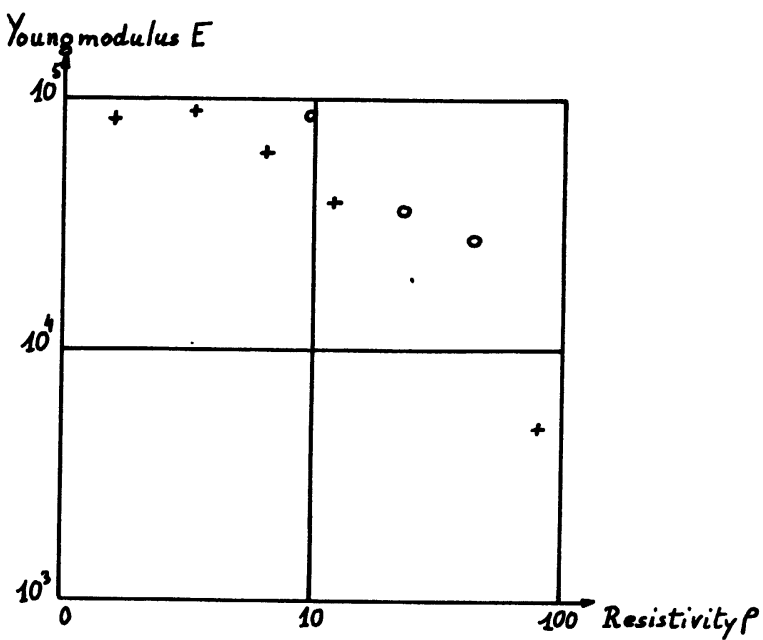

Fig. 7. - Young modulus versus resistivity in a Log-Log plot for short fibers armed gels. The concentration in fibers varies from 0 (right hand side of the plot) to $3.5 \%$ (left). The three circles correspond to a concentration of $1.5 \%$ (expected percolation threshold). Let us note that, however large is the dispersion of data in figure 6 , the elastic properties are very well correlated to the electrical ones.

than measured electrically and corresponding to a limit of more strongly entangled fibers. The increase seen in figure 5 would correspond to a precursory behaviour to be compared with the increase of the elasticity of soft plus hard spheres below a percolation threshold of the hard objects (see formula A.2). A somewhat similar effect is found in the work of Feng and Sen [9] in which they considered an incompletely connected triangular lattice of springs (central forces) without any bond-bending elasticity (see Appendix). If we consider that, at the electrical continuity threshold of the lattice, there is some freedom of rotation of two fibers in contact, rigidity is achieved at a higher threshold corresponding to a larger average number of contacts between fibers. Using an excluded volume argument developped in chapter 2.1, this would suggest a threshold for entangled fibers of the order of 2 to 3 times the electrical one if no further correlation between the orientations of neighbouring fibers is created by the mixing and when contacts are created. This seems to be the case at least in the case of sedimentating fibers (discussed below).

3.3 SETTLING-SEDIMENTING-FIBERS. - In order to isolate the effect of the entangled fibers from the matrix, we have studied the sediment formed in liquids whose density was adjustable and smaller than that of the fibers. By measuring the height of the sediment we deduce the volume fraction as a fonction of the length of the fibers. We have plotted our results obtained on sediments in water with different fiber lengths on the same plot of figure 8 as 


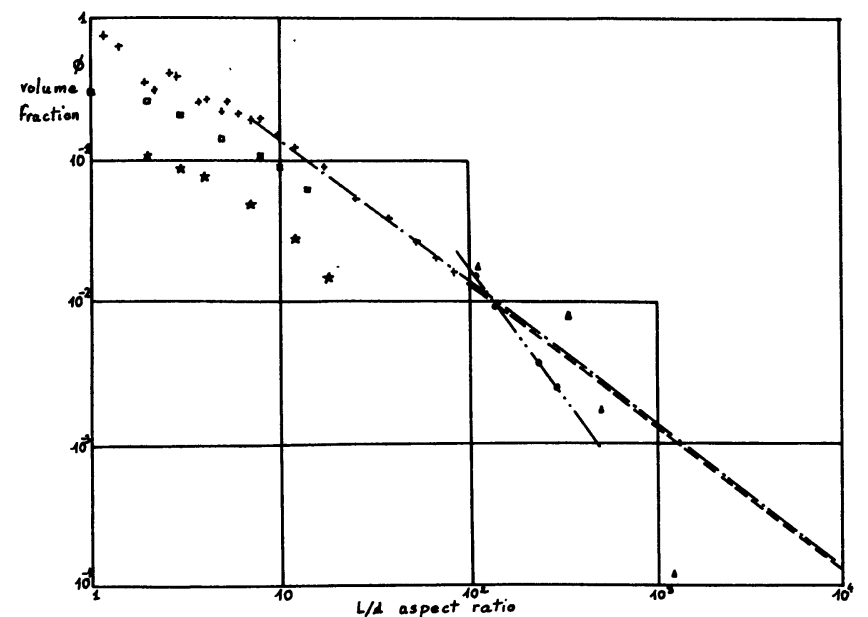

Fig. 8. - Percolation thresholds of randomly distribution fibers, for various aspect ratio (i.e. length over radius). These results have been obtained by very different methods : $\Delta$ Sedimentation limit ; $\bullet$ Experimental points obtained from araldite/fibers composites; + Numerical simulation of Balberg et al. [2] ; $\square$ Numerical simulation of Boissonade, Barreau, Carmona (3) ; * Numerical simulation of Vold [10] Sedimentation limit; $\longrightarrow$ from reference $\left[2^{\prime}\right]: \varphi_{\mathrm{c}} \propto(L / r)^{-1} ;-\ldots-$ from reference $\left[2^{\prime \prime}\right]$ : $\varphi_{\mathrm{c}}=2.65(L / r)^{-1}$.

the electrical threshold value determined on epoxyde composites. For an aspect ratio of $10^{2}$, the concentration of the sediment $\Phi_{c} \sim 1.5 \times 10^{-2}$ is of the same order as the critical concentration given by the electrical measurements and the calculation. The variation of $\Phi$ with the fiber length is comparable to that for the previous situations. This indicates that entanglement thresholds and electrical ones are closely related, as suggested in the discussion of 3.2). The higher values for sediment also suggest that a larger average number of contacts is involved in this later problem as expected. As a rough approximation, we can use the analysis of 2.2 to estimate an entangled threshold corresponding to an average of 4-5 contacts per fiber so as to ensure rigidity. However, once we have reached the connectivity threshold we have an «infinite » cluster of fibers. The addition of more fibers to the sample cannot be done in a neutral way. Interactions of new fibers with others already present, will introduce large correlations in the distribution and orientation of fibers, over large scales of distance (excluded volume effect). Therefore in any real system, we will have a partial ordering of bunches of fibers in contact. Such correlations also appears below threshold $\Phi_{c}$, but are small and localized. Experimentally, they appear as negligible, unless other physical processes exist which « memorize » the history of the array, like for example due to the high viscosity of the embedding medium.

In a more speculative way, we might even relate the increase in the scatter of the data (Fig. 5) around $\Phi_{c}$ as the sign of a transition towards a correlated phase which would exist above $\Phi_{c}$ (see Appendix).

We have also plotted numerical results obtained by Vold [10] in 1959 on the sedimentation of rods made out of a chain of aligned spheres in contact. If one corrects for the volume fraction of spheres in such a rod, one finds again a very good agreement with the percolation variation of $\Phi_{c}(L)$ in percolation problem.

\section{Conclusion and openings.}

The sequence of experiments described above is only preliminary and suggests the need for more detailed material studies to ascertain the connection between the random geometry of short fiber composites and their mechanical properties. The relation between the electrical continuity and the problem of entanglements deserves a full study. It would also be of large experimental importance to be able to characterize (and if possible to control) directly the geometry of the fiber arrangement.

On the practical side, the existence of an electrical percolation threshold at low fiber concentration (a few percent) is of some importance (conducting polymers, antistatic materials). After drying, the gel-fiber composites lead to fiber blocks extremely light, conducting and which can be machined. For such applications, one would probably need to shift from hydrophilic gels like polyacrylamide used here, to hydrophobic ones (polystyrene).

The problem of sediments made of entangled fibers is also of basic and practical interest in particular in relation with filtration processes. One would like to control at the same time the permeability of the array as well as the mechanical behaviour. The former experiments can be done using a sensitive differential « permeability meter » developed in our laboratory [11], or in centrifugation experiments, like those of Buscall [12], measuring the variation of thickness of sediments with the centrifugal force, should also give an indication on the percolation behaviour of such entangled systems. It would be of particular interest to see if the non-linear response which was predicted in Doi and Kuzuu [13] work in rigid polymeric rods, due to the increase of the number of active contacts with applied pressure is present in this problem.

\section{Appendix.}

The theory of percolation provides a description for the singular properties of a random homogeneous mixture made of elements of very different properties (conductors + insulators ; hard + soft materials) around the concentration threshold where a continous phase first appears across the material.

In particular the conductivity of a composite 
mixture varies above the critical concentration $\Phi=\Phi_{c}$ of conducting objects as

$$
\sigma(\Phi) \sim \sigma_{1}\left[\frac{\Phi-\Phi_{c}}{\Phi_{c}}\right]^{t} .
$$

For sufficiently regular distributions, the exponent $t$ which describes the "rate» of increase of $\sigma(\Phi)$ from zero is independent of the dimension of space :

$$
\text { for } \quad d=3 \quad t \simeq 2 \pm 0.1 .
$$

If the mixture is made of very good conductors (superconductors) in concentration $\Phi$ and of poor conductors, the conductance diverges as concentration threshold is approached from below as

$$
\sigma(\Phi) \sim \sigma_{2}\left[\frac{\Phi_{\mathrm{c}}-\Phi}{\Phi_{\mathrm{c}}}\right]^{-s}
$$

for

$$
d=3 \quad s \sim 0.8
$$

$\sigma_{1}\left(\sigma_{2}\right)$ is a constant of the order of the conductivity of the good (poor) conductor.

If the mixture is made of a mixture of good and poor conductors, the transition takes place around the same value $\Phi_{\mathrm{c}}$ but with a smooth variation between the conductivity characteristic of a superconductor-conductor mixture for low $\Phi$ (formula (A.2)) and that of an insulator-conductor mixture (formula (A.1)) above threshold. The range of cross-over between the two regimes is given by

$$
\varepsilon=\Delta \Phi / \Phi_{\mathrm{c}} \sim\left(\sigma_{2} / \sigma_{1}\right)^{1 /(s+t)} .
$$

The conductivity value at threshold is

$$
\sigma(\Phi) \sim \sigma_{1}\left(\sigma_{2} / \sigma_{1}\right)^{t /(t+s)} .
$$

The results can be summarized in a simple formula

$$
\sigma(\Phi)=\sigma_{1} h^{t(t+s)} F\left(\varepsilon / h^{1 /(t+s)}\right)
$$

where

$$
h=\sigma_{2} / \sigma_{1}
$$

$F(x)$ is an homogeneous function of the variable which is constructed in such a way that is should give the above results (A.1) to (A.4) when $h, \varepsilon \rightarrow 0$, for $\varepsilon>0$ and $\varepsilon<0$.

The idea to apply electrical percolation to the mechanical properties of heterogeneous materials was first suggested by de Gennes [14] for the rheology of the sol-gel transition [15]. It makes use of the geometric correspondance of the statistical objects in the two problems (a statistical distribution of finite clusters below $\Phi_{\mathrm{c}}$; a ramified and tortuous continuous percolation lattice paths above $\Phi_{\mathrm{c}}$ ). Using this geometrical analogy, it was suggested that the continuous increase of elasticity above $\Phi_{c}$ could be characterized by the same critical exponent $t$ as in expression (A.1) for the conducting insulating mixture problem. A correspondance was also established between the critical increase of elasticity of three dimensional mixtures of hard objects and soft ones below the percolation threshold of the hard objects and with the dielectric problem (formula (A.2)). These correspondances were verified experimentally on several classes of gels. However the above "scalar elasticity» correspondance with the scalar electrical percolation problem, has to be modified to take into account the vector character of the displacement and force fields (Webman [16], Roux and Guyon [17]). New sets of critical exponents are predicted. In particular the critical onset of elasticity is determined by the critical exponent $\tau \sim 4$ instead of $t$ in (A.1). The large value of the exponent is related to the extreme softness of the lattice near threshold due to easy angular deformation taking place over a singular correlation length $\xi \sim L \varepsilon^{-0.89}$ (A.6), which is the scale of the mesh size of the tenous percolation lattice.

Only very few model experiments on three dimensional model composite materials have attempted to characterize the critical mechanical percolation behaviour. In particular the work of Deptuck et al. [18] using weak sintered metals with a filling factor $\Phi=1-p \quad(p$ porosity $)$ above $\Phi \sim 10 \%$ have measured both the electrical conductivity and elastic moduli near threshold and found a critical behaviour of both quantities with exponents $t=2.15 \pm 0.25$, $\tau=3.8 \pm 0.5$. We also considered Buscall [12] results on sediments made with clay particles or polymer latex where the elastic bulk modulus is calculated from the compression of the sediment under centrifugation. From the dependence of the volume fraction with centrifugal forces we have obtained a critical mechanical exponent $\tau \sim 3.6$. Both results suggest indeed that the « scalar » elasticity does not apply to the system. On the other hand the values are consistent with « vector " models on lattices involving both compression and bond bending terms.

A last class of lattice models which may be relevent to the present studies deals with objects in contact, with only central force interaction and no angular elasticity $[9,19]$. They lead to a higher threshold than the connectivity (or conductivity) one because simple continuity is not enough to insure rigidity. Similarly we may think that random unconsolidated packings of objects in contact may exhibit a rigidity threshold larger than a connectivity one as suggested in the present work.

\section{Acknowledgments.}

We are very grateful to $\mathrm{C}$. Allain for suggesting the charged gel experiments and for discussions along this work. We also acknowledge B. Gautier Manuel, 
J. C. Charmet and M. Carmona for numerous discussions and advices along the work. Last but not least, we wish to thank M. Clement for his interest all along this work.

\section{References}

[1] Hashin, Z., J. Appl. Mech. 50 (1983) 481.

[2] Balberg, I., Binenbaum, N., Wagner, N., Phys. Rev. Lett. 52 (1984) 17 ;

Balberg, I., Anderson, C. H., Wagner, N., Phys. Rev. B 30 (84) 3939.

[3] Carmona, F., Barreau, F., Delaes, P., Canet, R., J. Physique Lett. 41 (1980) L-531.

[4] Gauthier Manuel, B., Thèse Paris (1986).

[5] De Gennes, P. G., The Physics of Liquid Crystals (Oxford Press, Clarendon, London) 1974, p. 36.

[6] Santalo, L. A., Integral Geometry and Geometric Probability (Addison Wesley) 1986.

[7] VAReille, J., Thèse Paris VI, 1986 and to be published.

[8] Treloar, L. R. G., The physics of rubber Elasticity (Pergamon) 1975.

[9] Feng, S., Sen, P. N., HAlperin, B. I., LobB, G. J., Phys. Rev. B 30 (1984) 5386 ;

Feng, S., Sen, P. N., Phys. Rev. Lett. 52 (1984) 216.
[10] Vold, M. J., J. Phys. Chem. 63 (1959) 1608.

[11] Amiel, C., Ambari, A., Gauthier Manuel, B., GuYon, E., Revue Phys. Appl. 21 (1986) 53.

[12] Buscall, R., Colloids Surfaces 5 (1982) 269.

[13] Kuzuu, N. Y., DoI, M., J. Polym. Sci. 18 (1980) 409.

[14] De Gennes, P. G., J. Physique Lett. 37 (1976) L-1.

[15] Allain, C., Gauthier Manuel, B., Jouhier, B., GUYON, E., in Percolation structure and processus, Ann. Isr. Soc., J. Adler, G. Deutcher, R. Zallen ed., p. 167.

[16] Webman, I., in Physics of finely divised matter, M. Daoud ed. (Springer Verlag) p. 180.

[17] Roux, S., GuYon, E., J. Physique Lett. 46 (1985) L999.

[18] Deptuck, D., Harrison, J. P., Zawadaski, P., Phys. Rev. Lett. 54 (1985) 913.

[19] Tremblay, A.-M. S., Breton, P., LemieuX, M. A., J. Physique Lett. 46 (1985) L-1. 